\title{
Association between Insulin-Like Growth Factor-1 and Uric Acid in Chinese Children and Adolescents with Idiopathic Short Stature: A Cross-Sectional Study
}

\author{
Panpan Wang (iD, Baolan Ji (iD, Qian Shao $(\mathbb{D}$, Mei Zhang $(\mathbb{D}$, and Bo Ban (iD \\ Department of Endocrinology, Affiliated Hospital of Jining Medical University, Jining, Shandong 272029, China \\ Correspondence should be addressed to Mei Zhang; zhangmei@mail.jnmc.edu.cn and Bo Ban; banbo2011@163.com
}

Received 26 September 2017; Revised 7 December 2017; Accepted 31 December 2017; Published 28 January 2018

Academic Editor: Flavia Prodam

Copyright (C) 2018 Panpan Wang et al. This is an open access article distributed under the Creative Commons Attribution License, which permits unrestricted use, distribution, and reproduction in any medium, provided the original work is properly cited.

\begin{abstract}
Objective. The aim of this study was to examine the relationship between insulin-like growth factor-1 (IGF-1) and serum uric acid (UA) in Chinese children and adolescents with idiopathic short stature (ISS). Methods. A cross-sectional study of 91 Chinese children and adolescents with ISS was performed. Anthropometric measurements and biochemical parameters were tested. The standard deviation score of IGF-1 (IGF-1 SDS) was calculated. Results. A univariate analysis displayed a significant positive correlation between IGF-1 SDS and UA $(P=0.004)$. In multivariate piecewise linear regression, the levels of IGF-1 SDS increased with the elevation of UA when UA was between $168 \mu \mathrm{mol} / \mathrm{L}$ and $301 \mu \mathrm{mol} / \mathrm{L}(\beta 0.010,95 \%$ CI $0.004-0.017 ; P=0.002)$. The levels of IGF-1 SDS decreased with the elevation of UA when UA was either less than $168 \mu \mathrm{mol} / \mathrm{L}(\beta-0.055,95 \%$ CI $-0.081--0.028$; $P<0.001)$ or more than $301 \mu \mathrm{mol} / \mathrm{L}(\beta-0.005,95 \% \mathrm{CI}-0.013-0.002 ; P=0.174)$. Conclusions. This study demonstrated a nonlinear relationship between IGF-1 and UA levels in Chinese children and adolescents with ISS. This finding suggests that either high or low levels of UA may have an adverse effect on IGF-1, whereas appropriate UA levels have a beneficial effect.
\end{abstract}

\section{Introduction}

The growth hormone-insulin-like growth factor-1 (GH-IGF1) axis is central to the regulation of growth and development. Low levels of GH or IGF-1 can result in short stature in childhood. Usually, children with idiopathic short stature (ISS) show normal IGF-1 levels, but in some other cases IGF-1 levels can be lower (e.g., GH receptor mutations or IGF acid-labile subunit gene variants) [1, 2]. Kim et al. have demonstrated that six months of GH treatment may not only increase the growth rate and improve height standard deviation score (SDS) but also increase IGF-1 and IGFbinding protein-3 (IGFBP-3) levels in children with ISS [3].

Related studies have shown that the generation of IGF-1 was influenced by diet and nutritional status $[4,5]$. Moreover, both the quantity and quality of foods intake may affect the production of IGF-1. Specifically, the levels of IGF-1 were positively correlated with the intake of red meat, fish, and oil, whereas the intake of carbohydrate foods had a negative effect on the levels of IGF-1 [6]. Additionally, starvation, semistarvation, fasting, and caloric restriction may lead to a decline in IGF-1 levels [7].

Uric acid (UA) was the final product of purine metabolism of nucleic acids. The majority of serum UA was freely filtered through the kidney glomeruli. According to preliminary estimates, approximately $90 \%$ of the filtered UA was reabsorbed by renal tubule [8], which implied that it may have a considerable physiological role in humans. Research showed that the levels of UA were influenced by a variety of internal and external factors including genetics and diet [9]. Therefore, as it was a metabolic product, the levels of UA could reflect a range of dietary intakes to some extent. In addition, studies have revealed that poor eating behaviors such as picky eating, partial eating, and even malnutrition are prevalent among children with short stature [10], which may affect the levels of UA. Previous studies on UA mainly focused on gout [11]. Recently, UA has been found to be correlated with many metabolic diseases such as obesity, metabolic syndrome, nonalcoholic fatty liver disease (NAFLD), type 2 diabetes, and cardiovascular diseases [12-16]. Regrettably, 
there was little related research on the relationship between IGF-1 and UA. Only Sesti et al. have demonstrated an inverse correlation between IGF-1 and UA levels in nondiabetic adults [17].

Until now, the association between IGF-1 levels and UA concentrations in children with ISS has not been studied. The aim of this study was to examine the relationship between IGF-1 and UA in Chinese children and adolescents with ISS.

\section{Subjects and Methods}

2.1. Study Subjects. We retrospectively reviewed the medical records of short-statured children and adolescents from the Department of Endocrinology, Affiliated Hospital of Jining Medical University, between January 2015 and December 2016. Ninety-one children and adolescents with ISS (68 males and 23 females) aged $10.1 \pm 3.8$ years were enrolled in this study. The subjects were selected based on the following inclusion criteria: short stature, a condition wherein the height of an individual was more than 2 SD scores (SDSs) below the corresponding mean height for a given age, sex, and population group; normal weight and height at birth; peak $\mathrm{GH}$ levels $>10 \mathrm{ng} / \mathrm{ml}$ in at least two $\mathrm{GH}$ stimulation tests. The exclusion criteria included the presence of chronic organic diseases, GH deficiency, chromosomal abnormalities, skeletal dysplasia, genetic metabolic diseases, thyroid dysfunction, and abnormal liver function, as well as the use of medication that interferes with $\mathrm{GH}$ secretion or function [18].

The study was approved by the Human Ethics Committee of the Affiliated Hospital of Jining Medical University (Shandong, China). All of the families of the patients were informed of the aims of the study, and informed consent was written and obtained from all patients of the subjects.

2.2. Anthropomorphic Measurements. Heights were measured by a specially designated individual using the same measuring instrument (produced by Nantong Best Industrial Co. Ltd., Jiangsu, China) in the morning with an allowable error range of $0.1 \mathrm{~cm}$. Height SDS was calculated based on normal range of Chinese children [19]. BMI was calculated as the ratio between body weight in kilograms and height in meters squared. The stage of puberty was assessed by physical examinations according to Tanner staging [20]. The following criteria were considered as prepuberty $[21,22]$ : boys with a testicular volume of less than $4 \mathrm{~mL}$ and no pubic hair and girls with no breast development and no pubic hair.

2.3. Laboratory Measurements. To assess GH secretion, Ldopa (Levodopa Tablets ${ }^{\circledR}$, He Feng, Guang Xi, China, body weight more than $30 \mathrm{~kg}$, $500 \mathrm{mg}$ of levodopa; less than $30 \mathrm{~kg}$, $250 \mathrm{mg}$ of levodopa) and insulin (Insulin Injection ${ }^{\circledR}$, Wan Bang, Jiang Su, China, $0.1 \mathrm{U} / \mathrm{kg}$ ) were administered orally or subcutaneously after overnight fasting. Blood samples were collected $0,30,60,90$, and $120 \mathrm{~min}$ later to obtain the serum $\mathrm{GH}$ concentration for each time point. $\mathrm{GH}$ was measured using a chemiluminescence method (ACCESS2, Beckman Coulter; USA) with an analytical sensitivity of $0.010 \mu \mathrm{g} / \mathrm{L}$. Serum IGF-1 and IGFBP-3 levels were measured by the chemiluminescence immunometric method (DPC

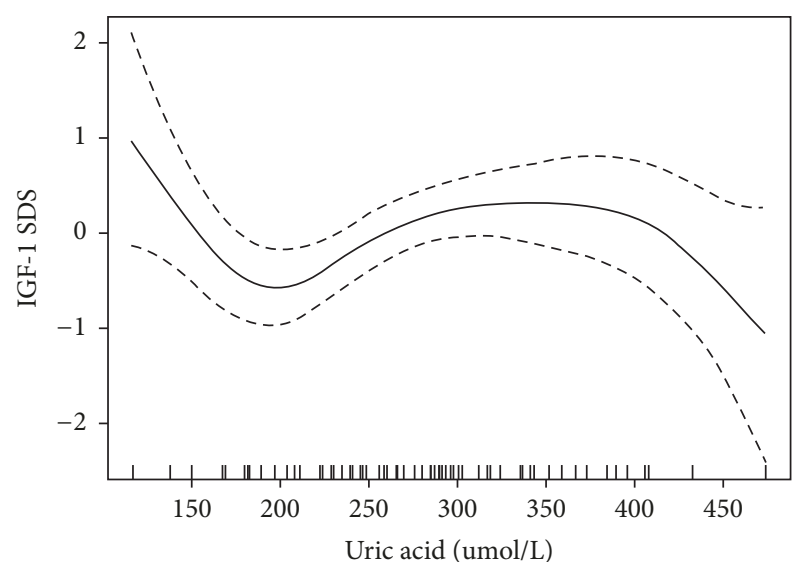

FIGURE 1: The relationship between IGF-1 SDS and UA by smooth curve fitting. Adjustment variables: body weight, pubertal stage, TG, LDL-C, Cr, and FPG. TG: triglyceride; LDL-C: low-density lipoprotein cholesterol; Cr: creatinine; FPG: fasting plasma glucose.

IMMULITE 1000 analyzer, SIEMENS, Germany) with intraand interassay CVs for IGF-1 of $3.0 \%$ and $6.2 \%$, respectively, and intra- and interassay CVs for IGFBP-3 of $4.4 \%$ and $6.6 \%$, respectively. Measures of liver function (including alanine aminotransferase (ALT), AST, and gamma-glutamyl transferase (GGT)), kidney function (including Cr, blood urea nitrogen (BUN), and UA), lipid profiles (including total cholesterol (TC), high-density lipoprotein cholesterol (HDLC), LDL-C, and triglycerides (TG)), and fasting plasma glucose (FPG) were tested by a biochemical autoanalyzer (Cobas c 702, Roche; Shanghai, China). Measures of thyroid function, including Free T3 (FT3), Free T4 (FT4), thyroidstimulating hormone (TSH), gonadotropin, cortisol rhythm, and adreno-cortico-tropic hormone (ACTH), were tested by a luminescence immunoassay system (Cobas e 602, Roche; Shanghai, China). The standard deviation score of IGF-1 (IGF-1 SDS) was calculated according to a previous study [23].

2.4. Statistical Analysis. All of the statistical analyses were performed with R statistical software (https://www.r-project .org) and EmpowerStats (http://www.empowerstats.com, X\& $\mathrm{Y}$ solutions, Inc. Boston MA). Normally distributed variables are expressed as the mean \pm standard deviation (SD), abnormally distributed variables are shown as the median (quartile), and non-parametric tests were used. Qualitative data are expressed as the numbers and percentage $(n, \%)$. First, we describe the demographic characteristics and biochemical values of the subjects (Table 1). Next, a univariate analysis model (Table 2) was used to examine whether UA and other anthropometrical and biochemical variables were correlated to IGF-1 SDS. Then, we explored the relationship between IGF-1 SDS and UA by smooth curve fitting after adjustment for potential confounders (Figure 1). Finally, we further performed a multivariate piecewise linear regression model to assess the independent correlation between IGF-1 SDS and UA according to smooth curve fitting (Table 3). $P$ values (two-tailed) below 0.05 were considered statistically significant. 
TABLE 1: Demographic characteristics and biochemical values of the subjects.

\begin{tabular}{|c|c|}
\hline Variables & All \\
\hline Number & 91 \\
\hline Sex (male $n, \%)$ & $68(74.7 \%)$ \\
\hline Age (years) & $10.1 \pm 3.8$ \\
\hline SBP (mmHg) & $107.3 \pm 12.5$ \\
\hline DBP (mmHg) & $63.9 \pm 10.1$ \\
\hline Height (cm) & $126.3 \pm 20.0$ \\
\hline Body weight (kg) & $27.6 \pm 10.9$ \\
\hline BMI $\left(\mathrm{kg} / \mathrm{m}^{2}\right)$ & $16.56 \pm 2.21$ \\
\hline IGF-1 (ng/ml) & $210.08 \pm 148.89$ \\
\hline IGF-1 SDS & $-0.65 \pm 1.19$ \\
\hline IGFBP-3 $(\mu \mathrm{g} / \mathrm{ml})$ & $4.74 \pm 1.35$ \\
\hline $\mathrm{Cr}(\mu \mathrm{mol} / \mathrm{L})$ & $38.70 \pm 10.93$ \\
\hline $\mathrm{BUN}(\mu \mathrm{mol} / \mathrm{L})$ & $4.86 \pm 3.13$ \\
\hline $\mathrm{UA}(\mu \mathrm{mol} / \mathrm{L})$ & $271.39 \pm 70.28$ \\
\hline $\mathrm{TC}(\mathrm{mmol} / \mathrm{L})$ & $3.64 \pm 0.69$ \\
\hline TG (mmol/L) & $0.58(0.48-0.86)$ \\
\hline HDL-C (mmol/L) & $1.32 \pm 0.26$ \\
\hline LDL-C (mmol/L) & $1.92 \pm 0.51$ \\
\hline VLDL-C (mmol/L) & $0.40 \pm 0.20$ \\
\hline $\operatorname{ALT}(\mathrm{U} / \mathrm{L})$ & $14.00(12.00-17.30)$ \\
\hline AST (U/L) & $25.92 \pm 5.34$ \\
\hline GGT (U/L) & $11.91 \pm 2.55$ \\
\hline $\mathrm{FPG}(\mathrm{mmol} / \mathrm{L})$ & $4.55 \pm 0.41$ \\
\hline \multicolumn{2}{|l|}{ Pubertal stage } \\
\hline In prepuberty $(n, \%)$ & $67(73.6 \%)$ \\
\hline In puberty $(n, \%)$ & $24(26.4 \%)$ \\
\hline
\end{tabular}

SBP: systolic blood pressure; DBP: diastolic blood pressure; BMI: body mass index; IGF-1: insulin-like growth factor-1; IGF-1 SDS: the standard deviation score of IGF-1; IGFBP-3: insulin-like growth factor-binding protein-3; Cr: creatinine; BUN: blood urea nitrogen; UA: uric acid; TC: total cholesterol; TG: triglyceride; HDL-C: high-density lipoprotein cholesterol; LDL-C: low-density lipoprotein cholesterol; VLDL-C: very low-density lipoprotein cholesterol; ALT: alanine aminotransferase; AST: aspartate transaminase; GGT: gamma-glutamyl transferase; FPG: fasting plasma glucose. Normal distribution of data was presented as mean \pm standard deviation; nonnormal distribution of data was presented as median (interquartile range) and was log-transformed (base 10) before parametric tests; the categorical data was expressed by the numbers and percentage $(n, \%)$.

\section{Results}

3.1. Clinical and Laboratory Characteristics of the Subjects. The clinical and laboratory characteristics of the subjects are shown in Table 1 . A total of 91 subjects ( $25.3 \%$ females) with a mean age of $10.1 \pm 3.8$ years were included in this study. The majority of the subjects were in prepuberty $(67,73.6 \%)$. The mean levels of IGF-1 SDS and UA were $-0.65 \pm 1.19$ and $271.39 \pm 70.28 \mu \mathrm{mol} / \mathrm{L}$, respectively.

3.2. Correlations between IGF-1 SDS and Anthropometrical and Biochemical Variables. Univariate analysis was performed to analyze the relationship between IGF-1 SDS and each variable. As shown in Table 2, there was a significant positive correlation between the IGF-1 SDS and age, height, body weight, BMI, Cr, puberty stage $(P<0.001)$, and UA $(P=0.004)$, whereas a significant negative correlation was found between the IGF-1 SDS and LDL-C $(P=0.015)$ and $\operatorname{AST}(P=0.001)$.

3.3. The Independent Correlation between IGF-1 SDS and UA by Multivariate Piecewise Linear Regression. Covariate screening was used to screen for possible confounders. The screening criteria included effect factors producing a $>10 \%$ change when introducing covariates in the basic model or eliminating covariates in the regression model. The results revealed that body weight, pubertal stage, Cr, LDL-C, and TG met the filter criteria. In addition, smooth curve fitting was performed after adjustment for all variables, and the resultant curve exhibited a three-stage change and two breakpoints (Figure 1). Namely, there was an inverse relationship between IGF-1 SDS and UA when UA was before the first point or after the second point. However, there was a positive relationship between IGF-1 SDS and UA when UA was between the two points. Then, threshold saturation effects were analyzed based on the curve in Table 3, and the data indicated that the inflection points were $168 \mu \mathrm{mol} / \mathrm{L}$ and $301 \mu \mathrm{mol} / \mathrm{L}$. Specifically, the IGF-1 SDS decreased with the elevation of UA when UA was less than $168 \mu \mathrm{mol} / \mathrm{L}(\beta-0.055$, $95 \% \mathrm{CI}-0.081--0.028 ; P<0.001)$ or more than $301 \mu \mathrm{mol} / \mathrm{L}$ $(\beta-0.005,95 \%$ CI $-0.013-0.002 ; P=0.174)$, whereas the IGF-1 SDS increased with the elevation of UA when UA was between $168 \mu \mathrm{mol} / \mathrm{L}$ and $301 \mu \mathrm{mol} / \mathrm{L}$ ( $\beta$ 0.010, 95\% CI 0.004-0.017; $P=0.002$ ).

\section{Discussion}

In this cross-sectional study, we demonstrated a nonlinear association between IGF-1 SDS and serum UA in Chinese children and adolescents with ISS. Namely, there was a positive relationship between IGF-1 SDS and UA when the concentration of UA was between $168 \mu \mathrm{mol} / \mathrm{L}$ and $301 \mu \mathrm{mol} / \mathrm{L}$. In contrast, there was an inverse relationship between IGF-1 SDS and UA when the concentration of UA was either less than $168 \mu \mathrm{mol} / \mathrm{L}$ or more than $301 \mu \mathrm{mol} / \mathrm{L}$.

UA is the end product of purine metabolism in humans. It is known that high levels of UA are associated with many metabolic diseases [11-16], whereas a recent report declared that extremely low levels of serum UA may induce endothelial dysfunction [24]. Thus, we infer that UA levels that were too high or too low may both have negative impacts on the human body. Our study indicated an inverse relationship between IGF-1 SDS and UA when the concentration of UA was less than $168 \mu \mathrm{mol} / \mathrm{L}$. However, there has been no research on the relationship between IGF-1 and the low levels of UA until now. Given the small sample size of this group, it is necessary to carry out related studies to explore the correlation between IGF-1 and low levels of UA and its potential mechanism. In addition, although the difference was not statistically significant, our research showed an inverse trend between IGF-1 SDS and UA when the concentration of UA was greater than $301 \mu \mathrm{mol} / \mathrm{L}$. This relationship was consistent with findings by Sesti et al. [17]. Furthermore, Sesti et al. noted that exposing human hepatoma cells to UA at a concentration 
TABLE 2: Correlations between IGF-1 SDS and anthropometrical and biochemical variables.

\begin{tabular}{|c|c|c|c|}
\hline Variables & $\beta$ & $(95 \% \mathrm{CI})$ & $P$ value \\
\hline Age (years) & 0.161 & $(0.103,0.219)$ & $<0.001$ \\
\hline Height $(\mathrm{cm})$ & 0.035 & $(0.025,0.046)$ & $<0.001$ \\
\hline Body weight (kg) & 0.072 & $(0.054,0.090)$ & $<0.001$ \\
\hline BMI $\left(\mathrm{kg} / \mathrm{m}^{2}\right)$ & 0.253 & $(0.150,0.357)$ & $<0.001$ \\
\hline $\mathrm{Cr}(\mu \mathrm{mol} / \mathrm{L})$ & 0.057 & $(0.037,0.076)$ & $<0.001$ \\
\hline $\mathrm{BUN}(\mu \mathrm{mol} / \mathrm{L})$ & 0.008 & $(-0.072,0.088)$ & 0.847 \\
\hline $\mathrm{UA}(\mu \mathrm{mol} / \mathrm{L})$ & 0.005 & $(0.002,0.009)$ & 0.004 \\
\hline $\mathrm{TC}(\mathrm{mmol} / \mathrm{L})$ & -0.384 & $(-0.790,0.022)$ & 0.068 \\
\hline TG (mmol/L) & 0.660 & $(-0.308,1.628)$ & 0.186 \\
\hline HDL-C (mmol/L) & -0.841 & $(-1.856,0.175)$ & 0.109 \\
\hline LDL-C (mmol/L) & -0.704 & $(-1.258,-0.150)$ & 0.015 \\
\hline VLDL-C (mmol/L) & 1.251 & $(-0.096,2.527)$ & 0.073 \\
\hline $\operatorname{ALT}(\mathrm{U} / \mathrm{L})$ & -0.022 & $(-0.078,0.034)$ & 0.435 \\
\hline AST (U/L) & -0.083 & $(-0.130,-0.036)$ & 0.001 \\
\hline FPG (mmol/L) & 0.145 & $(-0.496,0.785)$ & 0.659 \\
\hline \multicolumn{4}{|l|}{ Sex } \\
\hline Male & & 0 & \\
\hline Female & -0.567 & $(-1.184,0.049)$ & 0.075 \\
\hline \multicolumn{4}{|l|}{ Puberty stage } \\
\hline In prepuberty & & 0 & \\
\hline In puberty & 1.578 & $(1.096,2.016)$ & $<0.001$ \\
\hline
\end{tabular}

TABLE 3: The independent correlation between IGF-1 SDS and UA by multivariate piecewise linear regression.

\begin{tabular}{lccc}
\hline Inflection point of uric acid $(\mu \mathrm{mol} / \mathrm{L})$ & $\beta$ & $(95 \% \mathrm{CI})$ & $P$ value \\
\hline$<168$ & -0.055 & $(-0.081,-0.028)$ & $<0.001$ \\
$168 \sim 301$ & 0.010 & $(0.004,0.017)$ & 0.002 \\
$>301$ & -0.005 & $(-0.013,0.002)$ & 0.174 \\
\hline
\end{tabular}

Adjustment variables: body weight, pubertal stage, TG, LDL-C, Cr, and FPG; TG: triglyceride; LDL-C: low-density lipoprotein cholesterol; Cr: creatinine; FPG: fasting plasma glucose; $P<0.05$ is considered to be statistically significant.

of $300 \mu \mathrm{mol} / \mathrm{L}$ could induce the downregulation of $\mathrm{GH}$ stimulated IGF-1 expression [17]. The potential mechanism of this phenomenon was that UA may impair the ability of GH to stimulate JAK2-STAT5 signaling, which was the most important intracellular signaling pathway stimulating transcription of IGF-1 by inducing oxidative stress and posttranslational changes of proteins [25]. Additionally, Guler et al. showed that IGF-1 infusion for 6 days could induce an increase in glomerular filtration rate (GFR) and a decrease in the serum UA concentration in two healthy adult subjects [26].

Notably, this study also described a positive relationship between IGF-1 SDS and UA when the concentrations of UA were between $168 \mu \mathrm{mol} / \mathrm{L}$ and $301 \mu \mathrm{mol} / \mathrm{L}$, which was inconsistent with previous findings [17]. To the best of our knowledge, previous studies showed that UA levels are approximately 10 -fold higher in humans than in other mammals, which means that UA levels increased in the process of human evolution [27]. This implies that UA may play an important role in the human body, rather than simply serving as a waste product of purine metabolism. Indeed, the physiological effects of UA involve many aspects, including antioxidant effects, mediation of type 2 immune responses, resistance to parasites, defense of the nervous system, and autoimmune diseases [28]. As far as our findings were concerned, although the specific mechanism remained unclear, we considered that it may differ based on the study population: previous subjects were adults $[17,26]$, and in vivo and in vitro studies showed that UA had detrimental effects on IGF-1, whereas our study indicated that UA was positively associated with IGF-1 in children and adolescents with ISS. There were some differences between adults and children in their eating habits and diet types. Picky eaters, partial eclipse, and even malnutrition were prevalent among children with short stature. Observational data indicated that purine content was higher in fish, seafood, meat, and the internal organs of animals [29]. UA may be used as an indicator for evaluating the intake of those foods. Indeed, Larsson et al. indicated that greater dietary intake of protein, red meat, fish, and seafood showed a significant positive 
association with higher IGF-1 concentrations in healthy, well-nourished men [4]. Therefore, we hypothesized that a widespread phenomenon of picky eating and partial eating may lead to inadequate intake of those foods in short children, which may affect the levels of UA and IGF-1. Adequate intake of more purine-rich foods may help increase IGF1 levels, which was conducive to growth and development. In addition, because IGF-1 was a common indicator of the effectiveness of GH treatment, the effect of high-purine diet should be considered when using IGF-1 to assess the effectiveness of recombinant human growth hormone (rhGH) treatment.

Several potential limitations of this study should be considered. First, we failed to collect information about the diet of the subjects and did not perform a diet analysis of our study group. Therefore, we intend to design the dietary questionnaire to further analyze the effects of diet on IGF1. Second, the levels of serum UA were influenced by diet, metabolism, and other factors. Therefore, it is necessary to measure enzymes related to UA synthesis or decomposition, such as xanthine oxidase or adenosine deaminase. In addition, the present study did not measure urinary UA levels for 24 hours, which was a stable indicator to assess the excretion of UA. Third, the number of samples in this study was relatively small, especially in the group of UA $<168 \mu \mathrm{mol} / \mathrm{L}$ $(n=5)$. Therefore, we should further verify our results by expanding the sample size in any future studies. Finally, because of the study's cross-sectional nature, the present findings only exhibited a nonlinear relationship between IGF1 levels and the serum UA concentration. Prospective basic and clinical studies are thus required to further confirm causality.

In conclusion, in this present study, we described a nonlinear relationship between IGF-1 and UA levels in Chinese children and adolescents with ISS after adjusting for several confounders. This finding suggests that either high or low levels of UA may have an adverse effect on IGF-1, whereas appropriate UA levels have a beneficial effect on IGF-1.

\section{Disclosure}

Panpan Wang and Baolan Ji are co-first authors.

\section{Conflicts of Interest}

The authors declare that they have no conflicts of interest.

\section{Authors' Contributions}

Panpan Wang and Baolan Ji conducted data analysis and finalized the manuscript. They contributed equally to the studies and should be considered as co-first authors. Panpan Wang and Qian Shao participated in data collection. Bo Ban and Mei Zhang have contributed to the design of the study and should be considered as co-corresponding authors. All authors reviewed the paper.

\section{Acknowledgments}

This research was carried out at the Affiliated Hospital of Jining Medical University and was supported by grants from the Natural Science Foundation of Shandong Province (no. ZR2014HP058) and Health and Family Planning Commission of Shandong Province (no. 2014-032).

\section{References}

[1] K. Vairamani, L. Merjaneh, P. Casano-Sancho et al., "Novel Dominant-Negative GH Receptor Mutations Expands the Spectrum of GHI and IGF-I Deficiency," Journal of the Endocrine Society, vol. 1, no. 4, pp. 345-358, 2017.

[2] H. M. Domené, P. A. Scaglia, A. S. Martínez et al., "Heterozygous IGFALS gene variants in idiopathic short stature and normal children: Impact on height and the IGF system," Hormone Research in Paediatrics, vol. 80, no. 6, pp. 413-423, 2014.

[3] H.-S. Kim, S. W. Yang, H.-W. Yoo et al., "Efficacy of shortterm growth hormone treatment in prepubertal children with idiopathic short stature," Yonsei Medical Journal, vol. 55, no. 1, pp. 53-60, 2014.

[4] S. C. Larsson, K. Wolk, K. Brismar, and A. Wolk, "Association of diet with serum insulin-like growth factor I in middle-aged and elderly men," American Journal of Clinical Nutrition, vol. 81, no. 5, pp. 1163-1167, 2005.

[5] R. Stawerska, E. Czkwianianc, J. Smyczyńska, M. Hilczer, and A. Lewiński, "Nutritional status in short stature children is related to both ghrelin and insulin-like growth factor I concentrations," Journal of Pediatric Gastroenterology and Nutrition, vol. 64, no. 5, pp. 812-817, 2017.

[6] A. Juul, "Serum levels of insulin-like growth factor I and its binding proteins in health and disease," Growth Hormone \& IGF Research, vol. 13, no. 4, pp. 113-170, 2003.

[7] D. R. Clemmons, A. Klibanski, L. E. Underwood et al., "Reduction of plasma immunoreactive somatomedin C during fasting in humans," The Journal of Clinical Endocrinology \& Metabolism, vol. 53, no. 6, pp. 1247-1250, 1981.

[8] J. Maiuolo, F. Oppedisano, S. Gratteri, C. Muscoli, and V. Mollace, "Regulation of uric acid metabolism and excretion," International Journal of Cardiology, vol. 213, pp. 8-14, 2016.

[9] M. A. Beydoun, J.-A. Canas, M. T. Fanelli-Kuczmarski, S. M. Tajuddin, M. K. Evans, and A. B. Zonderman, "Genetic risk scores, sex and dietary factors interact to alter serum uric acid trajectory among African-American urban adults," British Journal of Nutrition, vol. 117, no. 5, pp. 686-697, 2017.

[10] M. O. Savage, "Insulin-like growth factors, nutrition and growth," World Review of Nutrition and Dietetics, vol. 106, pp. 52-59, 2013.

[11] F. Martinon, V. Pétrilli, A. Mayor, A. Tardivel, and J. Tschopp, "Gout-associated uric acid crystals activate the NALP3 inflammasome," Nature, vol. 440, no. 7081, pp. 237-241, 2006.

[12] M. Ishiro, R. Takaya, Y. Mori et al., "Association of uric acid with obesity and endothelial dysfunction in children and early adolescents," Annals of Nutrition and Metabolism, vol. 62, no. 2, pp. 169-176, 2013.

[13] A. F. Rubio-Guerra, H. Morales-López, A. K. Garro-Almendaro et al., "Circulating levels of uric acid and risk for metabolic syndrome," Current Diabetes Reviews, vol. 13, no. 1, pp. 87-90, 2017. 
[14] C.-Q. Liu, C.-M. He, N. Chen et al., "Serum uric acid is independently and linearly associated with risk of nonalcoholic fatty liver disease in obese Chinese adults," Scientific Reports, vol. 6, Article ID 38605, 2016.

[15] O. Lamacchia, A. Fontana, A. Pacilli et al., "On the nonlinear association between serum uric acid levels and allcause mortality rate in patients with type 2 diabetes mellitus," Atherosclerosis, vol. 260, pp. 20-26, 2017.

[16] E. Cheong, S. Ryu, J.-Y. Lee et al., "Association between serum uric acid and cardiovascular mortality and all-cause mortality," Journal of Hypertension, vol. 35, pp. S3-S9, 2017.

[17] G. Sesti, M. L. Hribal, T. Procopio et al., "Low circulating insulin-like growth factor-1 levels are associated with high serum uric acid in nondiabetic adult subjects," Nutrition, Metabolism \& Cardiovascular Diseases, vol. 24, no. 12, pp. 13651372, 2014.

[18] P. Cohen, A. D. Rogol, C. L. Deal et al., "Consensus statement on the diagnosis and treatment of children with idiopathic short stature: a summary of the Growth Hormone Research Society, the Lawson Wilkins Pediatric Endocrine Society, and the European Society for Paediatric Endocrinology Workshop," The Journal of Clinical Endocrinology \& Metabolism, vol. 93, no. 11, pp. 4210-4217, 2008.

[19] H. Li, C. Y. Ji, X. N. Zong, and Y. Q. Zhang, "Height and weight standardized growth charts for Chinese children and adolescents aged 0 to 18 years," Zhonghua er ke za zhi, vol. 47, no. 7, pp. 487-492, 2009.

[20] J. M. Tanner and R. H. Whitehouse, "Clinical longitudinal standards for height, weight, height velocity, weight velocity, and stages of puberty," Archives of Disease in Childhood, vol. 51, no. 3, pp. 170-179, 1976.

[21] W. A. Marshall and J. M. Tanner, "Variations in pattern of pubertal changes in girls," Archives of Disease in Childhood, vol. 44, no. 235, pp. 291-303, 1969.

[22] W. A. Marshall and J. M. Tanner, "Variations in the pattern of pubertal changes in boys," Archives of Disease in Childhood, vol. 45, no. 239, pp. 13-23, 1970.

[23] T. Isojima, A. Shimatsu, S. Yokoya et al., "Standardized centile curves and reference intervals of serum insulin-like growth factor-I (IGF-I) levels in a normal Japanese population using the LMS method," Endocrine Journal, vol. 59, no. 9, pp. 771-780, 2012.

[24] A. Oberbach, J. Neuhaus, N. Jehmlich et al., "A global proteome approach in uric acid stimulated human aortic endothelial cells revealed regulation of multiple major cellular pathways," International Journal of Cardiology, vol. 176, no. 3, pp. 746-752, 2014.

[25] C. P. Hawkes and A. Grimberg, "Insulin-Like Growth FactorI is a Marker for the Nutritional State," Pediatric endocrinology reviews : PER, vol. 13, no. 2, pp. 499-511, 2015.

[26] H.-P. Guler, C. Schmid, J. Zapf, and E. R. Froesch, "Effects of recombinant insulin-like growth factor I on insulin secretion and renal function in normal human subjects," Proceedings of the National Acadamy of Sciences of the United States of America, vol. 86, no. 8, pp. 2868-2872, 1989.

[27] I. A. Bobulescu and O. W. Moe, "Renal transport of uric acid: evolving concepts and uncertainties," Advances in Chronic Kidney Disease, vol. 19, no. 6, pp. 358-371, 2012.

[28] R. El Ridi and H. Tallima, "Physiological functions and pathogenic potential of uric acid: A review," Journal of Advanced Research, vol. 8, no. 5, pp. 487-493, 2017.
[29] H. K. Choi, S. Liu, and G. Curhan, "Intake of purine-rich foods, protein, and dairy products and relationship to serum levels of uric acid: the third national health and nutrition examination survey," Arthritis \& Rheumatology, vol. 52, no. 1, pp. 283-289, 2005. 


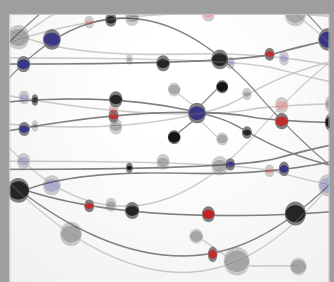

The Scientific World Journal
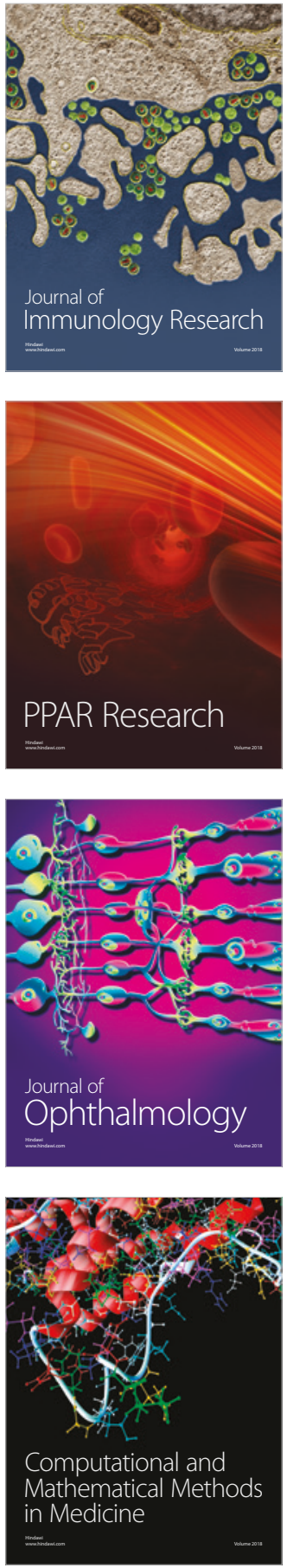

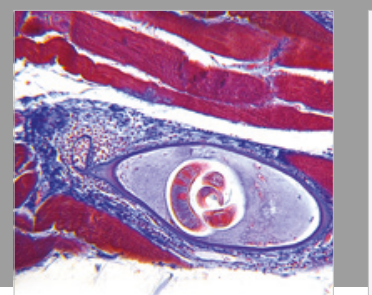

Gastroenterology Research and Practice

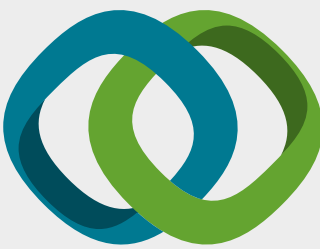

\section{Hindawi}

Submit your manuscripts at

www.hindawi.com
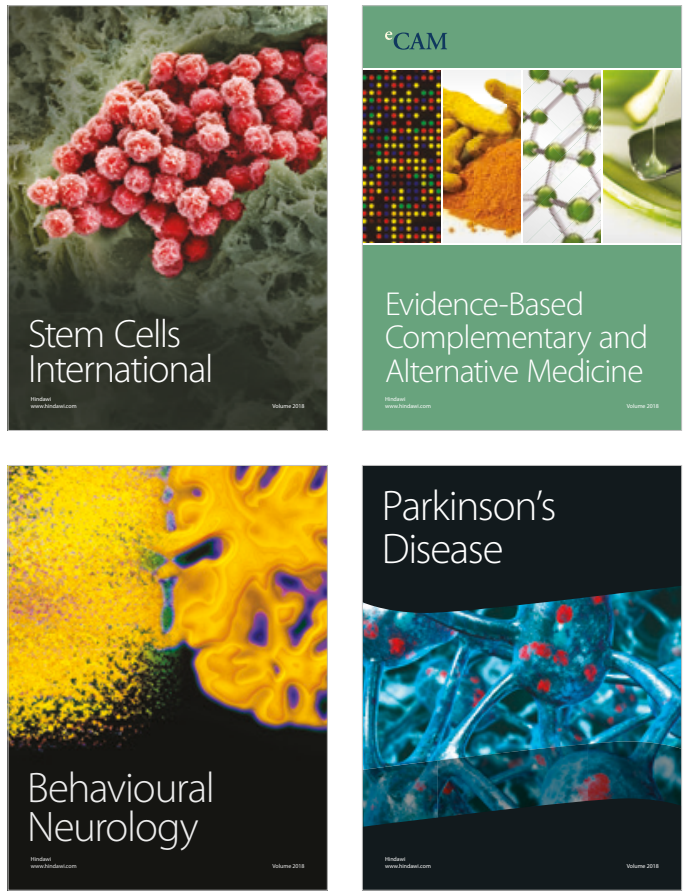

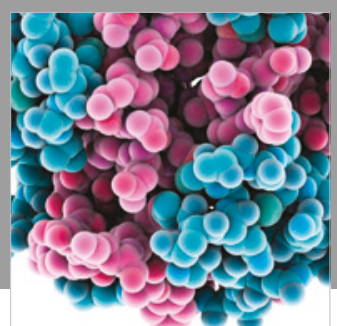

ournal of

Diabetes Research

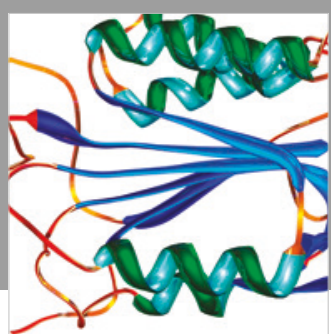

Disease Markers
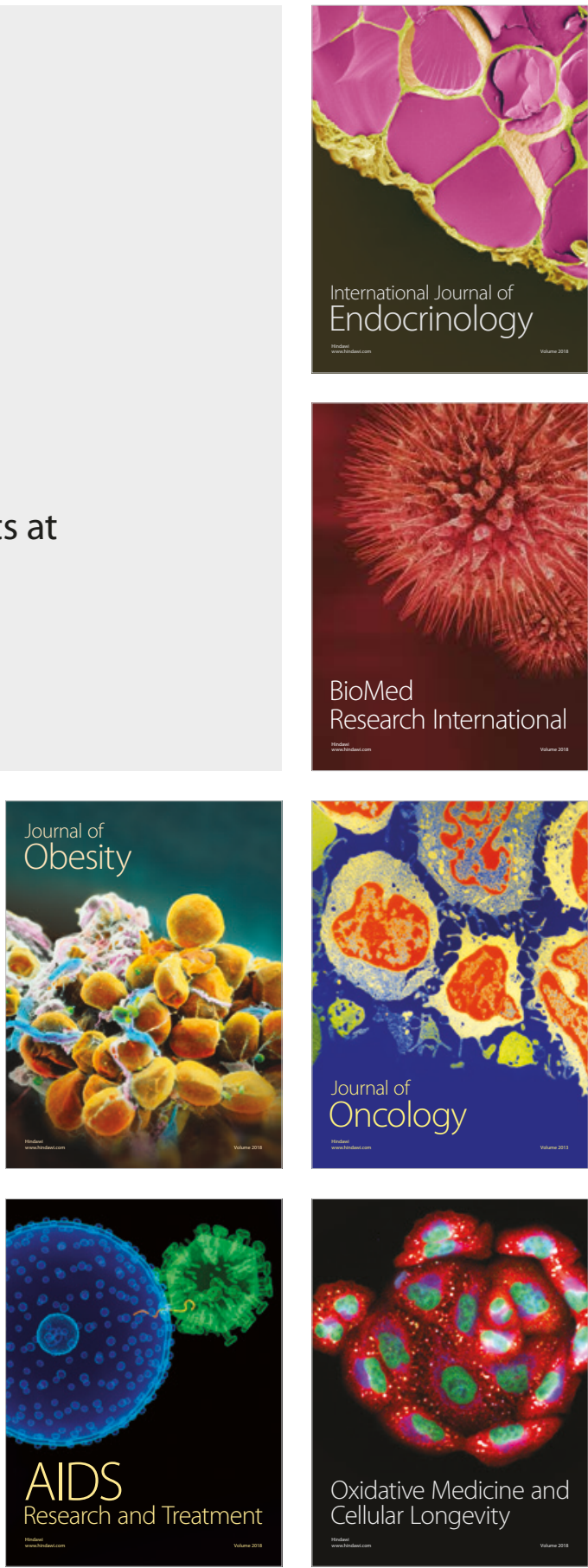